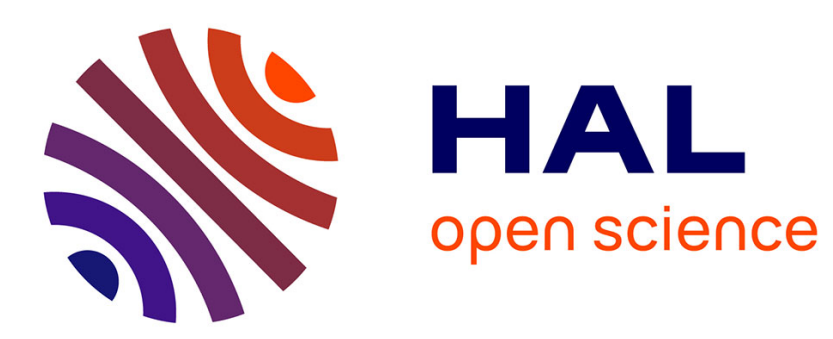

\title{
Modélisation de trajectoires sémantiques intégrant perspectives multiples et facteurs explicatifs
}

David Noël, Marlène Villanova-Oliver, Jérôme Gensel, Pierre Le Quéau

\section{To cite this version:}

David Noël, Marlène Villanova-Oliver, Jérôme Gensel, Pierre Le Quéau. Modélisation de trajectoires sémantiques intégrant perspectives multiples et facteurs explicatifs: Application aux trajectoires de vie. SAGEO Spatial Analysis and GEOmatics, Nov 2015, Hammamet, Tunisie. hal-01212778

\section{HAL Id: hal-01212778 \\ https://hal.science/hal-01212778}

Submitted on 7 Oct 2015

HAL is a multi-disciplinary open access archive for the deposit and dissemination of scientific research documents, whether they are published or not. The documents may come from teaching and research institutions in France or abroad, or from public or private research centers.
L'archive ouverte pluridisciplinaire HAL, est destinée au dépôt et à la diffusion de documents scientifiques de niveau recherche, publiés ou non, émanant des établissements d'enseignement et de recherche français ou étrangers, des laboratoires publics ou privés. 


\title{
Modélisation de trajectoires sémantiques intégrant perspectives multiples et facteurs explicatifs
}

\author{
Application aux trajectoires de vie
}

\author{
David Noël' ${ }^{1}$, Marlène Villanova-Oliver ${ }^{1}$, Jérôme Gensel ${ }^{1}$, Pierre \\ Le Quéau ${ }^{2}$
}

1. Equipe STeamer

Univ. Grenoble Alpes, LIG, F-38000 Grenoble, France

prenom.nom@imag.fr

2. Univ. Grenoble Alpes, PACTE, F-38000 Grenoble, France

pierre.le-queau@upmf-grenoble.fr

RESUME.

Nous proposons dans cet article un modèle de trajectoire sémantique qui supporte une certaine complexité dans la représentation des trajectoires. Nous considérons ainsi que la trajectoire est composée de multiples aspects qui constituent autant de perspectives selon lesquelles il est possible de l'observer. Nous permettons par ailleurs la représentation de facteurs explicatifs ayant influencé les épisodes constitutifs de cette trajectoire. Le cas d'application à l'origine de ce travail est présenté : celui des trajectoires de vie, avec un focus particulier sur la perspective résidentielle que cherchent à mieux appréhender les responsables politiques et les experts en aménagement urbain. Ceux-ci souhaitent en effet mieux comprendre les raisons des choix résidentiels des habitants dans le but d'adapter leurs décisions. Ces choix dépendent de facteurs concernant spécifiquement la résidence (type, surface...) ou son environnement (accessibilité du quartier, aménités disponibles...), mais ne peuvent être complètement appréhendés qu'en tenant compte des circonstances de vie de l'individu, par exemple familiales, professionnelles ou liées à la sphère des loisirs.

MoTS-CLES : Trajectoires sémantiques, trajectoires résidentielles, trajectoires de vies KEYWORDS: Semantic trajectories, Residential trajectories, Geospatial lifelines, Individual lifeline 


\section{Introduction}

Les métropoles doivent faire face à plusieurs challenges liés aux choix résidentiels de leurs habitants. Ces choix sont grandement influencés les phases de la vie personnelle des individus. Ainsi, les couples avec enfants privilégient un habitat éloigné des centres métropolitains tandis que ceux-ci attirent en plus grande proportion à la fois les jeunes nouvellement indépendants et les retraités (Bonvalet et Bringé, 2010). Ce constat illustre quelques-uns des problèmes les plus importants que les villes métropolitaines doivent résoudre. Cet exode des couples actifs avec enfants vers les zones périurbaines entraine pour les villes des périphéries des difficultés à fournir les aménités nécessaires (par exemple, des crèches et des écoles) mais aussi une augmentation des temps de trajets domicile-travail, générateur de pollution et de congestion du trafic. De même, dans les centres villes, on assiste à l'émergence de problèmes de cohabitation entre deux catégories d'âge aux modes de vies parfois différents. La diversification de la population des centres villes et de la périphérie des métropoles est donc un enjeu important et il est essentiel pour les décideurs politiques et les spécialistes de l'aménagement urbain d'acquérir une meilleure connaissance des dynamiques résidentielles. En particulier, il s'agit de mieux comprendre le processus de choix d'une résidence par un individu ou un ménage pour mieux s'y adapter.

Les raisons d'un choix résidentiel ne peuvent être appréhendées que dans le contexte d'une approche globale des trajectoires de vie des individus, c'est-à-dire en prenant en compte les aspects familiaux et professionnels, mais aussi ceux relevant de la sphère des loisirs et de tous les aspects de la vie qui sont déterminants dans le choix d'un individu ou d'un ménage donné. Le cadre de vie, notamment ce qui est lié aux caractéristiques de la résidence et de son voisinage, ainsi que d'autres éléments relevant des conditions économiques doivent également être envisagés (Lelévrier, 2007). Il s'agit plus précisément pour nous de tenir compte de la perception qu'ont les individus de ces différents éléments.

Ainsi pour étudier la trajectoire résidentielle d'un individu (i.e. quelle est la succession de ces choix résidentiels dans le temps et dans l'espace), il convient de la considérer comme une partie intégrée dans une vision plus large, celle de la trajectoire de vie de l'individu. Cette partie est une des perspectives multiples (déjà mises en évidence par (Thériault, 1999)) et interconnectées selon laquelle on peut observer la trajectoire de vie d'un individu. Notre objectif est de proposer un modèle générique capable de supporter cette complexité lié au caractère protéiforme d'une trajectoire et aux facteurs explicatifs que chacune des perspectives peut porter en elle pour expliquer l'évolution d'une autre perspective. Une illustration simple empruntée au cas des trajectoires de vie est le suivant: un déménagement (perspective résidentielle) peut-être expliqué par une mutation professionnelle (perspective professionnelle). Au delà de cet exemple trivial, un enjeu réside dans la représentation de la conjonction de plusieurs facteurs explicatifs de natures diverses (une mutation professionnelle peut avoir imposé un déménagement, un sentiment grandissant d'insécurité peut l'avoir initié). Un autre enjeu enfin relève de la possibilité de représenter, au delà des faits observés, (e.g. un déménagement avéré) 
des projets qui ont jalonné une trajectoire résidentielle idéale (e.g. le souhait de s'établir à terme à tel endroit dans tel type d'habitat).

Dans cet article, nous introduisons dans la section 2 les problématiques liés aux trajectoires de vies qui participent du cas d'application à l'origine de ce travail. Nous présentons dans la section 3 un bref état de l'art de la modélisation de trajectoires sémantiques et de trajectoires de vie, puis nous présentons dans la section 4 notre modèle intégrant multiples perspectives et facteurs explicatifs. Enfin, nous concluons et identifions des perspectives pour nos travaux futurs.

\section{Représenter et étudier des trajectoires de vie}

La Figure 1 représente un extrait de la trajectoire de vie d'un individu telle qu'elle a été travaillée précédemment par Thériault (Thériault, 1999). Elle est ici représentée comme une succession d'états qui sont caractérisés par valeurs des différents statuts ou attributs de cet individu («status» pour (Thériault, 1999)). Ces statuts peuvent être regroupés selon les divers aspects de la vie d'une personne (tels que résidentiel, familial, professionnel, loisirs, etc.), qui peuvent varier en fonction de chaque individu. Ces différents regroupements peuvent ainsi être considérés comme différentes trajectoires ciblant une thématique particulière. La trajectoire de vie est donc multidimensionnelle, chaque dimension correspondant à un focus « thématique » sur la trajectoire de vie. On parlera ainsi, par exemple, de trajectoire résidentielle représentée par les statuts est_propriétaire, loyer/prêt, type d'habitation et ville (cf. Figure 1). Tout statut, et par suite trajectoire, est intrinsèquement temporel(le) car lié(e) à une ligne de temps selon laquelle sont ordonnés les différents états de statuts.

Dans la Figure 1, seule la trajectoire résidentielle est une trajectoire spatiotemporelle car elle est constituée d'un statut contenant une information spatiale, ici la Ville de résidence, mais qui pourrait être considérée à une autre échelle spatiale, par exemple un quartier ou des coordonnées $\mathrm{x}, \mathrm{y}$. D'autres aspects de la vie de l'individu peuvent également contenir une composante spatiale, et être intéressant pour l'étude des trajectoires résidentielles, comme la localisation de son lieu de travail par exemple.

L'utilisation du terme de «trajectoire résidentielle » (Authier, 2010) ne fait pas référence qu'à l'aspect spatio-temporel de la vie résidentielle des individus. Pour les sociologues Yves Grafmeyer et Jean-Yves Authier (2011), parler de trajectoire est plus spécifique, que de décrire une simple évolution dans le temps et l'espace: "l'utilisation du terme de "trajectoire" plutôt que celui "d'itinéraire" ou de "mobilité", revient à suggérer qu'une série donnée de positions successives n'est pas le simple fait du hasard, mais s'enchaîne au contraire selon un ordre intelligible. » C'est là une partie de notre objectif : donner du sens à la trajectoire résidentielle, mais aussi comprendre les raisons sous-jacentes qui poussent les individus à changer de résidence, à quel moment de leur vie et pour quel (autre) espace. 


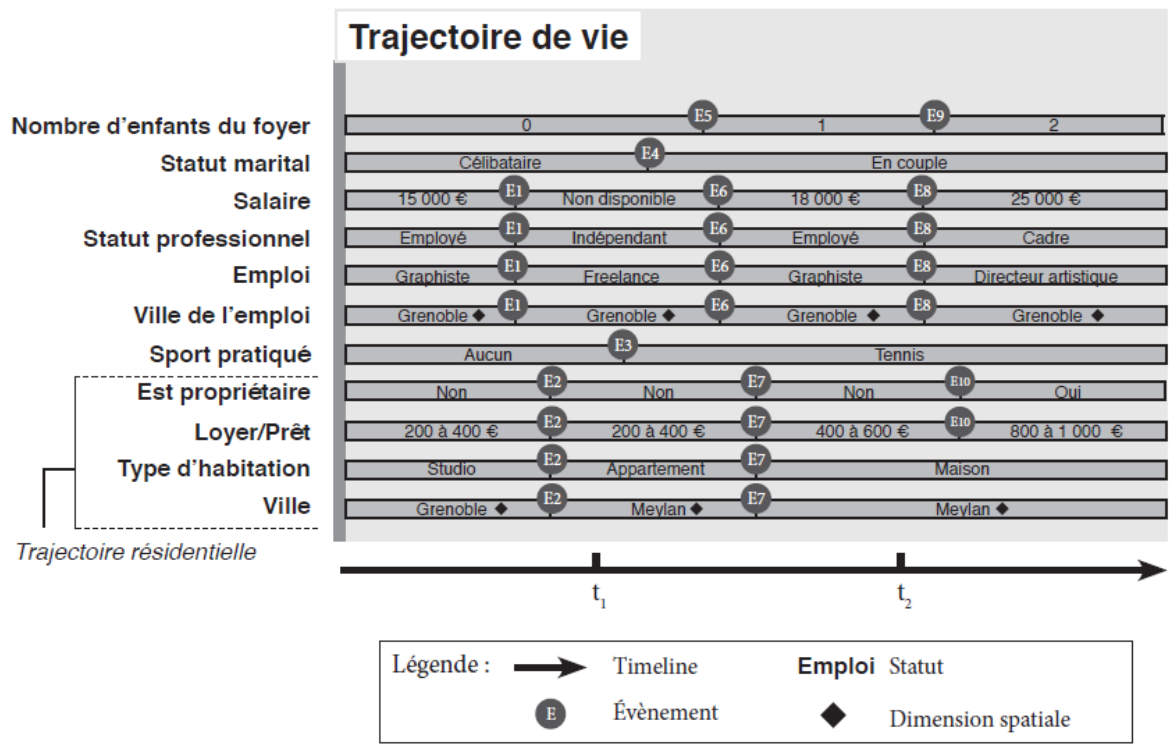

Figure 1 : extrait d'une trajectoire de vie. D'après Thériault (1999).

Le degré de maîtrise d'un individu sur sa trajectoire diffère, et il convient de mettre en évidence les conditions dans lesquelles chaque individu fait son/ses choix. Plus largement, il s'agit d'éliciter les facteurs explicatifs ou les combinaisons de facteurs qui ont contribué à modeler une trajectoire donnée. Ainsi, ce que nous cherchons ici à mettre évidence se rapproche de la notion de carrière telle qu'elle a été définie par Howard Becker (1985): "la carrière désigne les facteurs dont dépend la mobilité d'une position à l'autre, c'est-à-dire aussi bien les faits objectifs relevant de la structure sociale que les changements dans les perspectives, les motivations et les desseins des individus ». Dans le cas de la trajectoire résidentielle, ces facteurs sont d'une part à rechercher dans le contexte de la trajectoire de vie de l'individu. On parlera de facteurs internes dans le sens où ils sont directement liés aux caractéristiques propres d'une ou plusieurs perspectives de cette trajectoire de vie. D'autre part, les facteurs explicatifs peuvent être externes, c'est-à-dire non directement liés à cette trajectoire de vie en particulier. C'est le cas par exemple du marché de l'immobilier ou encore des caractéristiques de la résidence et de son environnement. Ces facteurs externes sont néanmoins à mettre en correspondance avec la trajectoire de vie. Par exemple, le fait que les prix de l'immobilier soient en hausse peut avoir un impact différent selon les trajectoires professionnelles des individus. Si de tels facteurs externes, pour lesquels il peut y avoir un certain consensus, peuvent être retenus comme explication plausible d'un événement, c'est bien le fait qu'ils soient explicitement mentionnés par l'individu comme facteur explicatif à un moment donné qui nous intéresse ici. 
La trajectoire de vie est complexe car elle est composée de nombreux attributs descriptifs, dont chacun peut se rapporter à un aspect différent de la vie de l'individu. L'organisation en perspectives facilite la compréhension et permet une meilleure lisibilité des caractéristiques importantes pour un focus thématique particulier. La représentation d'une trajectoire de vie est fortement dépendante du domaine d'étude et de l'objectif avec lequel on l'étudie. Dans le cas d'une étude en aménagement, le focus porte sur les attributs qui décrivent la trajectoire résidentielle, mais les facteurs explicatifs relèvent potentiellement d'autres thématiques. L'étude de la trajectoire de vie nécessite donc l'utilisation de référentiels adaptables, permettant de considérer les différentes parties de la trajectoire séparément mais aussi dans leurs interrelations. Ainsi, une modélisation pertinente devra permettre l'intégration des facteurs explicatifs de la trajectoire, qu'ils soient internes ou externes.

\section{Etat de l'art}

\subsection{Modélisation des trajectoires sémantiques}

De nombreux modèles de trajectoires sémantiques ont émergé ces dernières années, proposant différentes solutions pour enrichir les trajectoires spatiotemporelles. Il s'agit de passer de données GPS brutes, indiquant le déplacement d'un objet mobile, à une trajectoire contenant des informations utiles à sa description (cf. Figure 2). Ces informations peuvent, par exemple, être liées à la nature de l'objet mobile, la nature du mode de transport, ou encore sur les lieux traversés. Dans la Figure 2, l'objet mobile qui nous intéresse est une personne, et grâce à la sémantique ajoutée à la trajectoire on comprend qu'elle s'est rendue à son lieu de travail en vélo, et qu'elle est partie ensuite en voiture pour se rendre dans un magasin, des informations qui n'étaient pas contenues dans la trajectoire brute. Plus largement, toute information susceptible de donner du sens à une trajectoire peut être incluse dans un modèle de trajectoire sémantique. La modélisation des trajectoires sémantiques (Alvares \& al., 2007a) permet donc d'enrichir des données de mobilités brutes qui se présentent habituellement sous forme d'une trajectoire spatio-temporelle formée par une suite de triplets (latitude, longitude, temps), avec des informations susceptibles d'y apporter du sens.

La plupart des modélisations de trajectoires sémantiques repose sur les différents découpages proposés pour les trajectoires spatio-temporelles. En effet, en s'inspirant de la «time-geography » (Hägerstrand, 1970), des chercheurs ont modélisé les trajectoires spatio-temporelles grâce à des périodes de déplacements et des périodes d'activité (Zheni, 2008), ou encore de mouvements et d'arrêts (Spaccapietra, 2008). Ces structurations sont ensuite utilisées pour apporter les informations relatives à la trajectoire, comme le mode de transport lors d'un déplacement, ou la nature d'une activité.

Les approches explorées pour la modélisation de trajectoires sémantiques reposent principalement sur des types de données (Zheni, 2008) ou des ontologies (Baglioni, 2008). 


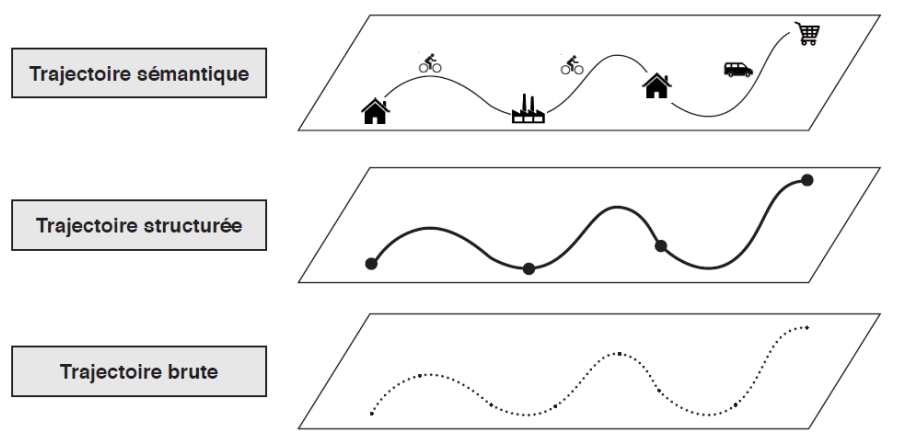

Figure 2 : De la trajectoire brute à la trajectoire sémantique, d'après Yan et Spaccapietra (2009)

\subsection{La modélisation de trajectoires de vie}

Les travaux conduits par Marius Thériault font référence dans le domaine de la modélisation de trajectoire de vie. Le modèle spatio-temporel pour l'analyse des trajectoires de vies (Thériault \& al, 1999) repose sur trois trajectoires différentes résidentielle, familiale et professionnelle. Chacune de ces trajectoires est modélisée conceptuellement par des épisodes - des statuts stables pendant un intervalle de temps -, et des événements qui viennent altérer un ou plusieurs de ces statuts. Le modèle conceptuel repose sur une approche relationnelle. Plus tard, le modèle sera modifié de façon à permettre de déterminer la probabilité qu'un événement survienne dans certaines conditions dans une trajectoire de vie (Thériault $\&$ al., 2002). Les conditions du choix résidentiel sont également abordées par (Vandermissen et al, 2009) avec une emphase particulière sur le rôle joué par les changements d'emploi qui interviennent dans la vie professionnelle des individus. Les modèles qui sont proposés dans ces travaux sont centrés sur l'aspect temporel des choix résidentiels : on s'intéresse davantage aux raisons pour lesquelles les individus vont déménager à un moment donné (en fonction de leurs circonstances de vie) qu'aux raisons pour lesquelles ils choisissent le lieu où ils vont emménager.

Les approches précédemment citées ont clairement posé les fondements conceptuels sur lesquels nous nous appuyons pour la représentation des trajectoires de vie. Nos travaux visent toutefois à enrichir cette représentation par un modèle de trajectoires sémantiques permettant la prise en compte de multiples perspectives, y compris relevant de projets et non d'observations avérées, et intégrant les différents facteurs explicatifs d'une trajectoire. 


\section{Un modèle pour les trajectoires de vie}

\subsection{L'utilisation de points de vue}

Pour traiter la question des différentes perspectives selon lesquelles est observée une trajectoire, nous nous appuyons sur la notion de point de vue ${ }^{1}$ (voir (Marino, 1993)). Un point de vue représente une perception parmi d'autres qu'un observateur a d'une entité étudiée. Le point de vue est constitué d'un sous-ensemble des attributs décrivant l'entité qui comprend l'attribut - ou les attributs - qui forme(nt) son identifiant ainsi qu'un sous ensemble des autres attributs. L'identifiant de l'entité est donc toujours présent dans un point de vue (ou visible de ce point de vue). Il n'y a pas forcément disjonction entre les points de vue, des attributs pouvant participer à plusieurs d'entre eux.

Conformément à la conception de point de vue la plus répandue (correspondant à la notion de vue dans les systèmes de gestion de bases de données), nous considérons que l'entité est unique. L'union des points de vue forme une vision complète (tous les attributs sont représentés) et cohérente (tous les attributs ont la même valeur dans chaque point de vue) de l'entité.

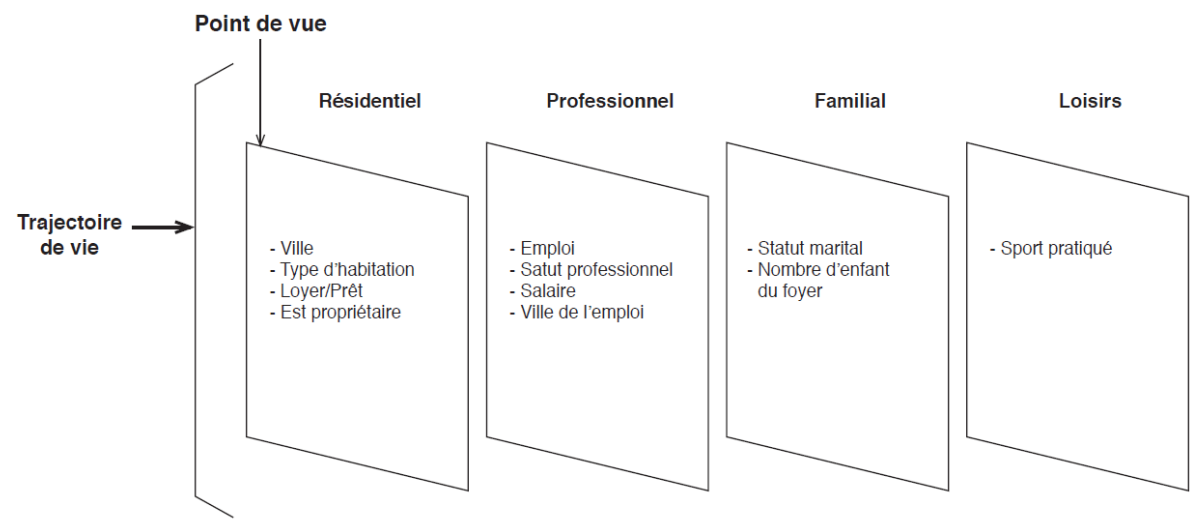

Figure 3 : Illustration de la notion de point de vue

La Figure 3 présente un exemple de quatre points de vue selon lesquels peut être observée la trajectoire d'une personne. Ces points de vue sont adaptables à chaque personne et/ou au cours du temps, soit par ajout d'un nouvel attribut dans un point de vue, soit par ajout d'un nouveau point de vue. Dans notre cas d'étude par exemple, les trois points de vue « résidentiel », « familial » et «professionnel » sont essentiels car ce sont des aspects de la vie qu'il est incontournable de prendre en compte pour trouver les facteurs explicatifs de la trajectoire résidentielle. D'autres

${ }^{1}$ Perspective et point de vue sont considérés comme des synonymes dans ce travail. 
points de vue peuvent cependant apparaitre en fonction des centres d'intérêt de l'individu étudié, tel que les loisirs, qui peuvent en effet, également influer sur les choix résidentiels.

Faute de place, nous ne présentons pas en détail la modélisation pour laquelle nous optons. Celle-ci repose sur le modèle de connaissances à objets multi-points de vue TROPES (Marino, 1993) qui intègre notamment une structuration de la connaissance dans un arbre de classes associé à chaque point de vue. Par exemple, dans le point de vue «résidentiel», une hiérarchie de classes peut être construite pour refléter les profils résidentiels des individus (tels les propriétaires aisés d'un appartement neuf en centre ville), en considérant les valeurs possibles pour les différents attributs constitutifs du point de vue. Cette approche est particulièrement intéressante car elle est associée à un mécanisme de classification des instances au sein de chaque point de vue qui permet par ailleurs d'opérer des comparaisons entre les trajectoires des individus. Nous définissons dans les sections suivantes les concepts clés de notre proposition.

\subsection{La structuration de la trajectoire de vie}

Notre approche associe les conceptions du temps de Leibniz et de Newton, le premier considérant le temps comme une succession d'événements, et le second comme un flux mesurable en valeur absolue. En effet, nous considérons l'évolution du temps comme une succession d'évènements, mais sans pour autant renoncer à le mesurer, grâce aux unités dont nous disposons (année, jour, heure...). Notre approche intègre les considérations portées par Claramunt et Thériault (Claramunt \& Thériault, 1995) (Claramunt, 1998) dans leurs travaux sur la gestion du temps dans les systèmes d'informations géographiques.

Le premier enjeu est de définir la granularité temporelle adaptée à la modélisation de la trajectoire de vie. Pour (Jensen, 1998), un chronon est une durée de temps minimum, la plus petite unité temporelle considérée pour marquer temporellement un événement. Dans le cas de la trajectoire de vie, un chronon pertinent peut être le jour, cette unité permettant de situer des événements marquants avec une précision qui fait sens pour un individu (comme la date d'un mariage, de la naissance d'un enfant, etc.). A l'échelle d'une vie, il est cependant utile de retenir un chronon d'une granularité moins fine telle que le mois ou l'année pour situer un événement pour lequel la précision en termes de jour est impossible (oubli de la date précise) ou non requise (situer quand a débuté le projet de déménager n'est pas forcément au jour près). Le modèle doit donc supporter des degrés de précision différents dans la datation.

Notre modèle de trajectoire de vie est composé d'épisodes qui décrivent l'état stable d'une personne considérée selon un point de vue particulier. L'état d'un point de vue est considéré comme stable quand les valeurs des attributs qui le composent restent inchangées au cours du temps. Ce choix de modélisation impose de bien choisir l'information à représenter (quels sont les attributs pertinents) mais aussi de déterminer le degré de précision pertinent. Ainsi, concernant par exemple le loyer d'un logement, ce n'est pas le montant exact qui est intéressant mais plutôt une 
fourchette de valeurs. Cela permet d'une part de catégoriser l'information (loyer modéré, élevé, etc.) et présente l'avantage d'en assurer une certaine stabilité. Il est en effet inutile pour l'objectif général (mieux appréhender les trajectoires résidentielles) d'enregistrer un nouvel épisode car le loyer a augmenté faiblement.

Sont également représentés les événements qui correspondent à quelque chose qui survient et qui peut être observé selon (Abler, 1971). L'événement marque généralement le passage d'un état stable à un autre dans un point de vue (début et fin d'épisodes).

Définition d'un épisode. Un épisode correspond à l'état stable d'une personne observée d'un certain point de vue pendant un intervalle de temps. La durée de l'épisode correspond à un intervalle de temps $A$ compris entre deux instants $\mathrm{t}_{\mathrm{s}}$ et $\mathrm{t}_{\mathrm{e}}$ (tel que $t_{s}<t_{e}$ ). Chacun de ces deux instants peut correspondre à un événement dans la trajectoire.

Ainsi, un épisode Epi de la trajectoire d'une personne $P$ observée d'un point de vue $i$ est composé de l'identifiant $k_{P}$ de $P$ et des attributs $a_{j}, \ldots, a_{k}$, et est valable sur un intervalle de temps $\left[t_{s}, t_{e}\right]$. On note :

$$
\operatorname{P.i.Epi}\left(k_{P}, a_{j}, \ldots, a_{k}\right)\left[t_{s}, t_{e}\right]
$$

Dans l'exemple de la Figure 1, représentant la trajectoire de vie de Léo un épisode valable du 20/05/2000 аи 30/06/2004 est noté ainsi :

$$
\begin{gathered}
\text { Léo.résidentiel.Epi }\left(k_{P}=123\right. \text {, Ville="Grenoble", Habitation="Appartement", } \\
\text { Loyer }=[200 ; 400])[20 / 05 / 2000,30 / 06 / 2004]
\end{gathered}
$$

Entre les épisodes, une relation d'ordre non stricte peut être établie grâce à l'algèbre d'Allen (Allen, 1983).

Définition d'un événement. Un événement est rattaché à un point de vue $i$ pour un individu $P$, a un identifiant $k_{E v}$, un nom name_evt, et est associé à un chronon $t$ pertinent pour le phénomène observé (ce qui permet de le situer temporellement). On note :

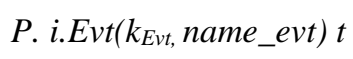

Par exemple un événement déménagement survenu le 30/06/2004 dans la trajectoire de Léo est noté dans le point de vue résidentiel:

$$
\text { Léo.résidentiel.Evt }\left(K_{E v t}=1\right. \text {, name_evt="déménagement") 30/06/2004 }
$$

L'attribut name_evt est choisi dans un vocabulaire contrôlé et indique la nature de l'événement. Une ontologie structurant l'ensemble de ce vocabulaire, spécifique à chaque point de vue, est en cours de définition.

Définition d'une trajectoire. La trajectoire de vie $T$ d'une personne $P$ est constituée de l'union des points de vue $i$, chacun étant défini par un ensemble d'événements et d'épisodes.

$$
P . T=\{U \text { P.i } \text { pour } i \in\{\text { Résidentiel, Professionnel, Familial, ... }\}
$$


Une trajectoire simplifiée comprenant un seul point de vue $i$ composé de deux événements et de deux épisodes dont le dernier est en encore en cours peut donc être notée de la manière suivante :

$$
\begin{gathered}
\text { P.T.i }=\left\{\text { P.i.Evt }\left(k_{E v t,}, \text { name_evt }\right) t_{1} ; P . i . E p i\left(k_{P}, a_{j}, \ldots, a_{k}\right)\left[t_{1}, t_{2}\right] ; P . i . E v t\left(k_{E v t,},\right.\right. \\
\text { name_evt } \left.) t_{2} ; P . i . E p i\left(k_{P}, a_{j}, \ldots, a_{k}\right)\left[t_{2}, a u j\right]\right\}
\end{gathered}
$$

Avec $t_{1}<t_{12}, t_{2}<a u j$, auj= date du jour et une valeur parmi les attributs $\left(a_{j}, \ldots\right.$, $a_{k}$ diffère entre P.i.Epi $\left(k_{P}, a_{j}, \ldots, a_{k}\right)\left[t_{1}, t_{2}\right]$ et P.i.Epi $\left(k_{P}, a_{j}, \ldots, a_{k}\right)\left[t_{2}\right.$, auj].

\subsection{L'intégration des facteurs explicatifs}

La trajectoire de vie d'une personne est donc marquée par des événements qui viennent modifier son état d'un point de vue donné. A ces événements peuvent être associés des facteurs explicatifs, qu'il nous semble essentiel de modéliser.

Les facteurs explicatifs peuvent être internes ou externes, c'est-à-dire qu'ils peuvent soit être relatifs aux circonstances de vie de l'individu soit dépendre de facteurs qui n'y sont pas liés directement. Par exemple, les facteurs internes pourront être liés à la naissance d'un enfant ou à un changement de situation professionnelle. Il apparaît donc que dans notre modèle, les événements eux-mêmes peuvent être des facteurs explicatifs. Ainsi, l'événement «changement d'emploi » peut être un facteur explicatif de l'événement «déménagement». Les facteurs explicatifs de la trajectoire résidentielle de l'individu peuvent également être trouvés dans des conditions de l'environnement (économique, social, etc.) dans lequel évolue l'individu. C'est par exemple le cas du prix du marché de l'immobilier qui peut limiter l'achat ou le déménagement dans un quartier donné. Nous ne souhaitons modéliser ces facteurs externes que dans la mesure où les individus se sentent impactés par ceux-ci, ce qui se traduit par l'ajout dans la trajectoire d'un événement exprimant un ressenti. L'événement « Naissance d'un sentiment d'insécurité » est un exemple ${ }^{2}$.

Un facteur explicatif $\mathrm{F}$ est à associer à l'événement qu'il explique. Chaque événement pouvant être expliqué par plusieurs facteurs, on étend la définition donnée précédemment pour un événement et on note :

$$
\begin{gathered}
\text { P.i.Evt }\left(k_{E v t}, \text { name_evt, }\left\{F_{1}, \ldots, F_{n}\right) t\right. \\
\text { avec } F_{j}\left(k_{E x p}, \text { description }\right)
\end{gathered}
$$

Chaque facteur explicatif $F_{j}$ associe l'identifiant $k_{E x p}$ d'un événement (l'explication) et un attribut description qui permet de préciser l'influence de l'événement pour le point de vue $i$ auquel appartient l'événement $k_{E v t}$ expliqué.

\footnotetext{
2 Conformément aux définitions précédemment données, cela aura pour effet de créer un nouvel épisode en raison du changement de valeur d'un attribut dédié au sentiment de sécurité qu'il convient de représenter dans ce contexte.
} 


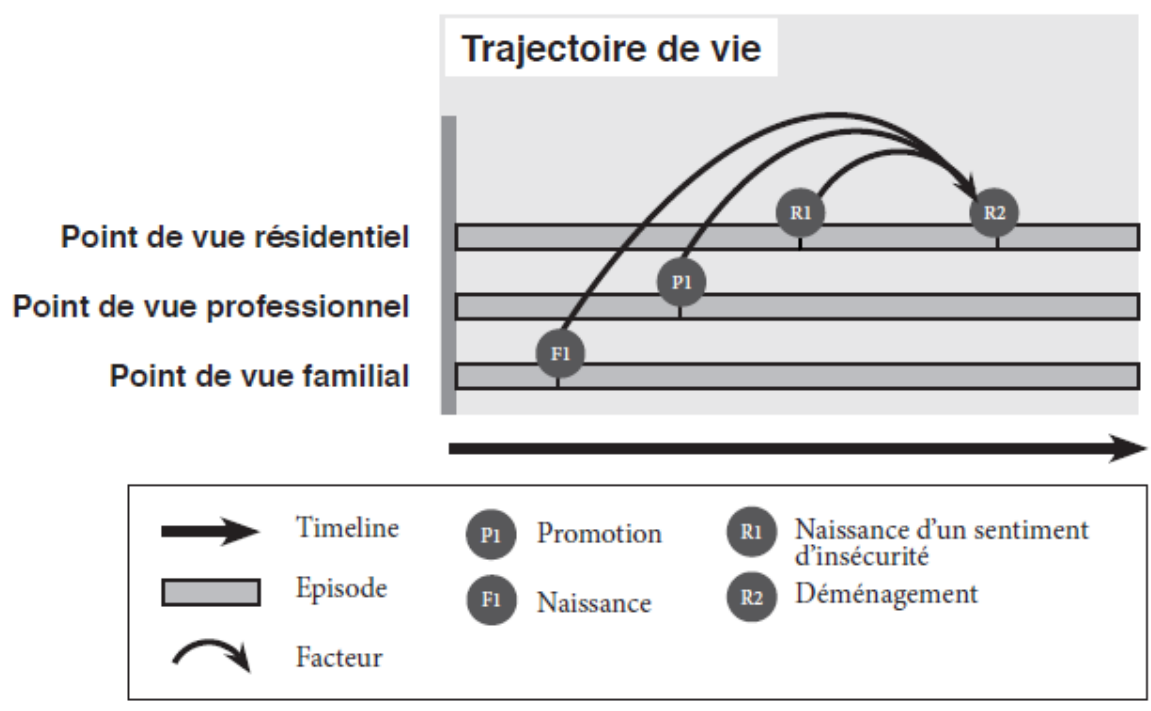

Figure 4 : Les facteurs explicatifs d'un événement. Les valeurs données dans les pastilles correspondent aux identifiants des événements

Dans l'exemple de la Figure 4 représentant un extrait de la trajectoire de vie de Mila, il existe trois facteurs explicatifs de l'événement R2 Déménagement

Mila.résidentiel.Evt $\left(k_{E v t}=R 2\right.$, name_evt="déménagement", $\left.\left\{F_{1}, F_{2}, F_{3}\right\}\right)$ 20/05/2014

et ceux-ci peuvent être modélisés de la façon suivante. :

$F_{1}(R 1$, "Les effractions dans mon quartier en mars 2015 m'ont incité à partir")

$F_{2}(P 1$, "L'augmentation de mon salaire me permet un loyer supérieur")

$$
F_{3}(F 1, \text { "Il nous fallait une chambre de plus") }
$$

On note que les facteurs explicatifs peuvent être inter-points de vue ou intrapoint de vue, c'est-à-dire qu'ils peuvent dépendre d'événements survenus au sein du même point de vue que l'événement qu'ils expliquent, ou bien d'événements survenus dans un autre point de vue (cf. Figure 4).

\section{Exploitation du modèle}

Dans cette partie, nous présentons comment peut être exploité le modèle que nous avons proposé. Dans un premier temps nous montrons en quoi les concepts définis jusqu'ici peuvent être utilisés pour enrichir encore la représentation des trajectoires de vie en modélisant les aspirations d'un individu. Puis, nous illustrons 
par quelques exemples les possibilités d'exploitation des connaissances que le modèle permet d'envisager.

\subsection{Trajectoire avérée et aspirations}

Les facteurs explicatifs des choix résidentiels, qu'ils relèvent de faits avérés ou de ressentis de l'individu ne suffisent pas toujours à expliquer sa trajectoire. Les projets des individus, ou plus largement, leurs envies ou leurs aspirations doivent également être pris en compte et donc modélisés. Les concepts présentés précédemment (épisodes, événements et facteurs explicatifs) peuvent être exploités dans ce but. Nous pouvons ainsi représenter non plus les trajectoires avérées des individus mais les projets qu'ils ont fait ou font pour le futur.

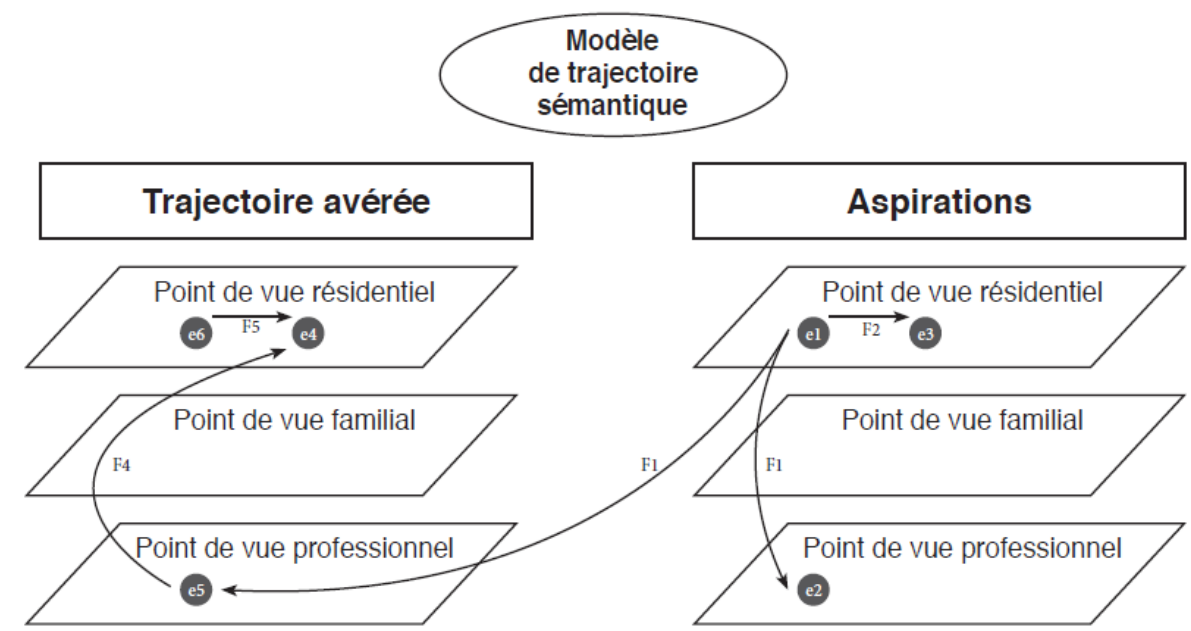

Figure 5 : Trajectoire avérée et aspirations (représentation des événements et des facteurs explicatifs)

Ainsi, il est possible de représenter les projets résidentiels ou professionnels que Léo a faits par le passé alors qu'il habitait Grenoble, et les raisons de ces choix (les événements et leurs facteurs explicatifs sont représentés sur la Figure 5) :

Léo.projet_résidentiel.Epi $\left(k_{P}=35\right.$, Ville="Bron", Habitation="Appartement") [2000, 2005]

Léo.projet_professionnel.Epi $\left(k_{P}=35\right.$,Ville_Emp="Lyon", Emploi="Etudiant") [2000, 2005]

Léo.projet_résidentiel.Evt $\left(K_{E v t}=e 1\right.$, name_evt="Offre") avril2000

Léo.projet_professionnel.Evt $\left(K_{E v t}=e 2\right.$, name_evt="IntégrerEcole", F1) avril2000 
Léo.projet_résidentiel.Evt $\left(K_{E v t}=e 3\right.$, name_evt="Déménagement", F2) août2000

F1 (e1, "Ma tante possédait un appartement à Bron et me l'avait proposé donc le choix de l'école à Lyon était envisageable")

\section{F2 (e1, "L'appartement à Bron était disponible à partir d'août")}

Ceci traduirait que Léo avait envisagé en avril 2000 d'intégrer une Ecole à Lyon pour y étudier parce que l'appartement de sa tante lui avait été proposé (et non l'inverse, il n'a pas envisagé de déménager à Bron parce qu'il intégrait cette école). Le déménagement était envisageable en aout 2000 compte tenu de l'offre de sa tante.

$\mathrm{Si}$, par ailleurs, il est avéré que Léo a bien intégré l'Ecole de Lyon à la rentrée de septembre 2000, mais que sa tante a finalement vendu son appartement en juin 2000, il a dû trouver un autre logement dans la métropole lyonnaise.

$$
\begin{aligned}
& \text { Léo.résidentiel.Epi }\left(k_{P}=35\right. \text {, Ville ="Villeurbanne", Habitation="Studio") [2000, } \\
& \text { 2001] } \\
& \text { Léo.professionnel.Epi }\left(k_{P}=35\right. \text {, Ville_Emp="Lyon", Emploi="Etudiant") [2000, } \\
& \text { 2005] } \\
& \text { Léo.résidentiel.Evt }\left(K_{E v t}=e 4,\right. \text { name_evt="Déménagement", F4, F5) sept2000 }
\end{aligned}
$$

Léo.professionnel.Evt $\left(K_{E v t}=e 5\right.$, name_evt="Intégrer Ecole", F1) sept2000

Léo.résidentiel.Evt $\left(K_{E v t}=e 6\right.$, name_evt="Pas de solution de logement") juin2000

$$
\text { F4 (e5, "J'ai intégré l'Ecole à Lyon") }
$$

F5 (e6, "Ma tante a vendu son appartement, ")

F1 (e1, "Ma tante possédait un appartement à Bron et me l'avait proposé donc le choix de l'école à Lyon était envisageable")

Cet exemple illustre qu'il est possible d'exploiter le modèle pour représenter deux types de trajectoires, celle avérée (i.e. où et quand sont effectivement passés les individus) et celle qui aurait pu exister ou à laquelle l'individu aspire. Les deux peuvent être mises en parallèle pour mesurer des écarts et les analyser. En particulier, des cas intéressants peuvent apparaître, comme celui lié à l'événement $e 1$ exprimé à l'origine dans une trajectoire envisagée mais qui impacte au final la trajectoire avérée.

\subsection{Requêtage du modèle}

Le modèle peut être exploité grâce à des entrées temporelles, spatiales et/ou thématiques moyennant le recours à un langage de requête adapté au formalisme d'implémentation du modèle. Concernant la dimension temporelle, il peut par exemple être utilisé pour connaître la situation d'un ou plusieurs individus à un instant $t$ ou pendant un intervalle de temps $\left[t_{1}, t_{2}\right]$. Sur le plan spatial, il est possible de savoir quels lieux (selon la granularité retenue pour les attributs spatiaux du 
modèle) font partie de la trajectoire des individus. De telles requêtes peuvent concerner la localisation des résidences ou des lieux de travail et leur éventuelle proximité. Naturellement, des requêtes sont aussi possibles sur le plan spatiotemporel ou encore thématique.

Le modèle permet également de connaître les facteurs explicatifs liés à la mobilité résidentielle pour un individu particulier ou pour un groupe d'individus. Il est par exemple possible de s'intéresser à un événement précis de la trajectoire et de rechercher ses causes.

De même des requêtes alliant dimensions temporelles, ou spatiales, avec des facteurs explicatifs sont possibles. Pour le point de vue résidentiel, on peut ainsi savoir pour un déménagement donné à quelle date émerge le premier facteur explicatif. Il est ainsi possible de définir une période de latence, qui correspond à un intervalle de temps pendant laquelle un individu est susceptible de former le projet de déménager.

\section{Conclusion et perspectives}

Nous avons proposé un modèle original de trajectoires sémantiques intégrant de multiples perspectives ainsi que des facteurs explicatifs. Nous nous sommes appuyés sur le cas d'application des trajectoires de vie pour lequel nous avons montré l'utilité de ces deux notions. L'utilisation du concept de point de vue permet une grande adaptabilité en fonction des informations étudiées, en plus d'une classification à la fois dans le temps et entre les individus. De plus, l'intégration de modèle de facteurs explicatifs nous permet de mieux comprendre les circonstances des déménagements des individus, qu'elles dépendent de facteurs externes ou internes à leurs trajectoires.

Nous concevons actuellement une algèbre et des algorithmes qui permettront de manipuler ce modèle dont l'implémentation selon les principes du modèle TROPES est en cours. Nous nous concentrons particulièrement sur la possibilité d'inférer des facteurs explicatifs des trajectoires, qui mettront en évidence des facteurs clés.

Pour anticiper sur l'exploitation du modèle, il convient de noter qu'il devra pouvoir être alimenté par les individus eux-mêmes (au moyen d'une application dédiée, ou encore pour partie par moissonnage des réseaux sociaux), mais que des techniques d'inférence, en s'appuyant par exemple sur des individus prototypiques, devront aussi permettre de construire une certaine forme de connaissance relatives aux trajectoires de vie.

\section{Références}

Abler, R., Adams, J. S., \& Gould, P. (1971). Spatial organization: the geographer's view of the world.

Allen, J. F. (1983). Maintaining knowledge about temporal intervals. Communications of the $A C M, 26(11), 832-843$. 
Allen, J. F. (1984). Towards a general theory of action and time. Artificial intelligence, 23(2), 123-154.

Alvares, L. O., Bogorny, V., Kuijpers, B., de Macelo, J. A. F., Moelans, B., \& Palma, A. T. (2007). Towards semantic trajectory knowledge discovery. Data Mining and Knowledge Discovery.

Alvares, L. O., Bogorny, V., Kuijpers, B., de Macedo, J. A. F., Moelans, B., \& Vaisman, A. (2007). A model for enriching trajectories with semantic geographical information. In Proceedings of the 15th annual ACM international symposium on Advances in geographic information systems (p. 22). ACM.

Authier, J. Y., Bidet, J., Collet, A., Gilbert, P., \& Steinmetz, H. (2010). État des lieux sur les trajectoires résidentielles.

Baglioni, M., Macedo, J., Renso, C., \& Wachowicz, M. (2008). An ontology-based approach for the semantic modelling and reasoning on trajectories. In Advances in Conceptual Modeling-Challenges and Opportunities (pp. 344-353). Springer Berlin Heidelberg.

Becker, H. S. (1985). Outsiders: études de sociologie de la déviance. Editions Métailié.

Bonvalet, C. ; Bringé, A. (2010), « Les trajectoires géographiques des Franciliens depuis leur départ de chez les parents », in C. Bonvalet \& E. Lelièvre (eds) De la famille à l'entourage, Paris, Ined.

Claramunt, C., Parent, C., \& Thériault, M. (1998). Design patterns for spatio-temporal processes. In Data Mining and Reverse Engineering (pp. 455-475). Springer US.

Claramunt, C., \& Thériault, M. (1995). Managing time in GIS an event-oriented approach. In Recent Advances in Temporal Databases (pp. 23-42). Springer London.

Zheni, D., Frihida, A., Ghezala, H. B., \& Claramunt, C. (2009). A semantic approach for the modeling of trajectories in space and time. In Advances in Conceptual ModelingChallenging Perspectives (pp. 347-356). Springer Berlin Heidelberg.

Jensen, C. S., Dyreson, C. E., Böhlen, M., Clifford, J., Elmasri, R., Gadia, S. ... \& Wiederhold, G. (1998). The consensus glossary of temporal database concepts-february 1998 version (pp. 367-405). Springer Berlin Heidelberg.

Goodchild, M. F. (2007). Citizens as sensors: the world of volunteered geography. GeoJournal, 69(4), 211-221.

Grafmeyer, Y., \& Authier, J. Y. (2011). Sociologie urbaine. Armand colin.

Hägerstrand, T. (1970). What about people in regional science? Papers in regional science, 24(1), 7-24.

Lelévrier, C. (2007), Mobilités et trajectoires résidentielles des ménages dans trois opérations de rénovation urbaine en Île-de-France, rapport final de recherche, PUCA.

Marino Drews, O. (1993). Raisonnement classificatoire dans une représentation à objets multi-points de vue.

Spaccapietra, S., Parent, C., Damiani, M. L., de Macedo, J. A., Porto, F., \& Vangenot, C. (2008). A conceptual view on trajectories. Data \& knowledge engineering, 65(1), 126146. 
Thériault, M., Séguin, A. M., Aubé, Y., \& Villeneuve, P. Y. (1999). A spatio-temporal data model for analysing personal biographies. In Database and Expert Systems Applications, 1999. Proceedings. Tenth International Workshop on (pp. 410-418). IEEE.

Thériault, M., Claramunt, C., Séguin, A. M., \& Villeneuve, P. (2002). Temporal GIS and statistical modelling of personal lifelines. In Advances in Spatial Data Handling (pp. 433 449). Springer Berlin Heidelberg.

Yan, Z., \& Spaccapietra, S. (2009, August). Towards Semantic Trajectory Data Analysis: A Conceptual and Computational Approach. In VLDB PhD Workshop.

Vandersmissen, M. H., Séguin, A. M., Thériault, M., \& Claramunt, C. (2009). Modeling propensity to move after job change using event history analysis and temporal GIS. Journal of geographical systems, 11(1), 37-65. 